\title{
A Measurement of the Absolute Reactor Antineutrino Flux and Spectrum at Daya Bay
}

\author{
Fengpeng $A \mathrm{n}^{1, \mathrm{a}}$ on behalf of the Daya Bay Collaboration \\ ${ }^{1}$ Meilong Road 130, East China University of Science and Technology, Shanghai, 200237, China
}

\begin{abstract}
The Daya Bay Reactor Neutrino Experiment uses an array of eight underground detectors to study antineutrinos from six reactor cores with different baselines. Since the start of data-taking from late 2011, Daya Bay has collected the largest sample of reactor antineutrino events to date, and has made the most precise measurement of the neutrino oscillation parameters $\sin ^{2} 2 \theta_{13}$ and $\Delta m_{e e}^{2}$. Using the data from the four detectors in the near experimental halls, Daya Bay has made a high statistics measurement of the absolute reactor antineutrino flux and spectrum. In this paper we will present this measurement and its comparison to predictions based on different flux models.
\end{abstract}

\section{Introduction}

Reactor neutrino experiments played an important role in neutrino physics since the first experimental observation of antineutrinos by Reines and Cowan et al.[1]. In 2012 Daya Bay experiment observed non-zero neutrino mixing angle $\theta_{13}$ with a significance more than $5 \sigma$ [2], consistent with recent measurements from other experiments[3-6]. The near future reactor neutrino experiments are aiming to determine the neutrino mass hierarchy, improve the precision of neutrino mixing parameters measurement, and search for short baseline neutrino oscillation. In reactors, electron antineutrinos are emitted primarily from the fissions of four isotopes: ${ }^{235} \mathrm{U},{ }^{238} \mathrm{U},{ }^{239} \mathrm{Pu}$ and ${ }^{241} \mathrm{Pu}$. The modeling of the reactor antineutrino spectrum of the four isotopes are based on the measurements of the beta spectra at ILL Grenoble for ${ }^{235} \mathrm{U},{ }^{239} \mathrm{Pu}$ and ${ }^{241} \mathrm{Pu}$ [7-9], and the theoretical calculation from Vogel for ${ }^{238} \mathrm{U}$ [10], before 2011, and the predictions of the reactor antineutrino flux and spectrum based on the ILL+Vogel model agreed well with experiment data. In 2011, Huber and Muller carried out reevaluation of the antineutrino spectrum model with improved theoretical treatments $[11,12]$ and the predictions of the reactor antineutrino flux based on the Huber+Muller model was shown to be higher than the experimental data, implying a discrepancy which is commonly referred as the "Reactor Antineutrino Anomaly". Recent reactor neutrino experiments also observed an excess of detected events comparing to predictions in the antineutrino energy region of 5-7 MeV, raising the suspicion of the accuracy of existing reactor antineutrino prediction models. It is thus important to precisely measure the reactor antineutrino flux and spectrum and compare with model predictions. This paper will report the recent absolute reactor antineutrino flux and spectrum measurement of Daya Bay experiment based on 621 days of data taking, with more than 1.2 million antineutrino candidates collected from eight antineutrino detectors.

ae-mail: anfengpeng@gmail.com 


\section{The Daya Bay Reactor Neutrino Experiment}

The Daya Bay experiment is designed to precisely measure the neutrino mixing angle $\theta_{13}$ employing relative measurements between multiple detectors. The Daya Bay nuclear power complex is built at Daya Bay in southern China, about 55 kilometers to Hong Kong. As shown in Fig. 1 the power complex consists of three nuclear power plants (NPPs) and each NPP has a pair of reactor cores generating $2.9 \mathrm{GW}$ thermal power each. The reactor power plants are designed based on the French Framatome Advanced Nuclear Power three cooling loop design. The antineutrino detectors (ADs) were built in three underground experimental halls (EHs): EH1 and EH2 each consists of two identical $\mathrm{ADs}$ and $\mathrm{EH} 3$ has four ADs.

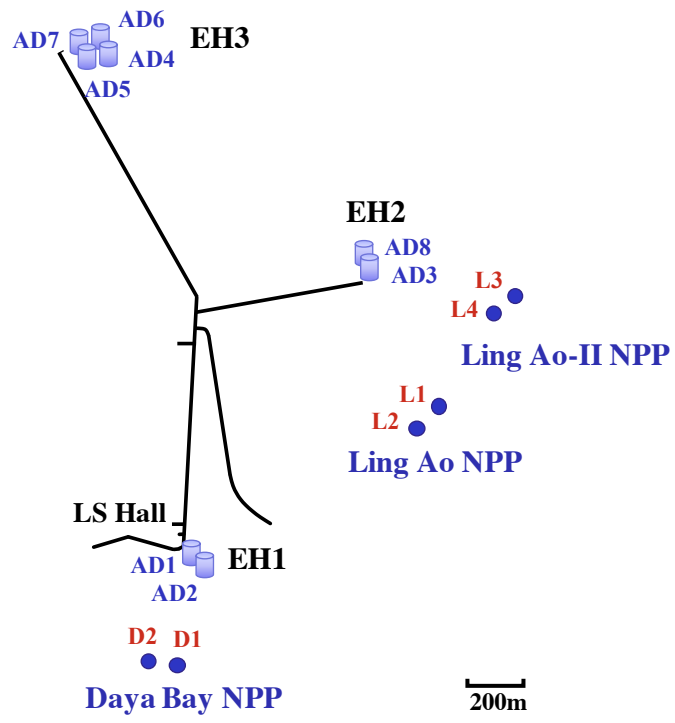

Figure 1: Layout of the full configuration of the Daya Bay experiment with eight antineutrino detectors (ADs) installed in three underground experimental halls (EHs). The dots represent reactor cores, labeled as D1, D2, L1, L2, L3 and L4.

The reactors are pure antineutrino sources. Almost all antineutrinos are produced from the fission of ${ }^{235} \mathrm{U},{ }^{238} \mathrm{U},{ }^{239} \mathrm{Pu}$ and ${ }^{241} \mathrm{Pu}$, fissions of other isotopes contribute less than $0.3 \%$. The reactor antineutrino spectrum with energy $E$ at time $t$ can be predicted by:

$$
\frac{d^{2} \phi(E, t)}{d E d t}=\frac{W_{t h}(t)}{\sum_{i} f_{i}(t) e_{i}} \sum_{i} f_{i}(t) S_{i}(E) c_{i}^{n e}(E, t)+S_{\mathrm{SNF}}(E, t)
$$

where $i$ is the index of the four isotopes, $W_{t h}$ is the thermal power of the reactor core measured by the systems of the NPP with $0.5 \%$ uncertainty, $f_{i}$ is the fission fraction of the isotope $i$ provided by the NPP using commercial reactor fuel evolution simulation package SCIENCE based on APOLLO2 code [13] as shown in Fig. 2, $e_{i}$ is the energy release per fission of isotope $i$ [14], $S_{i}(E)$ is the antineutrino spectrum of fission of isotope $i$ from prediction models, $c_{i}^{n e}$ is the correction factor for the non-equilibrium effects of long-lived fission fragments, and $S_{S N F}$ is the antineutrino contribution from the spent nuclear fuel. 


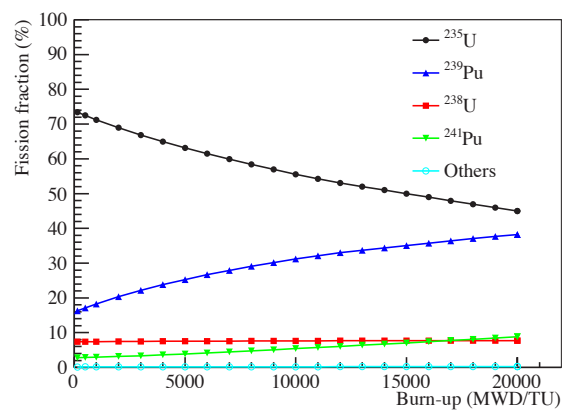

Figure 2: Fission fractions of isotopes in reactor core D1 as a function of cycle burn-up from a simulation of a complete refueling cycle. Other isotopes contribute less than $0.3 \%$.

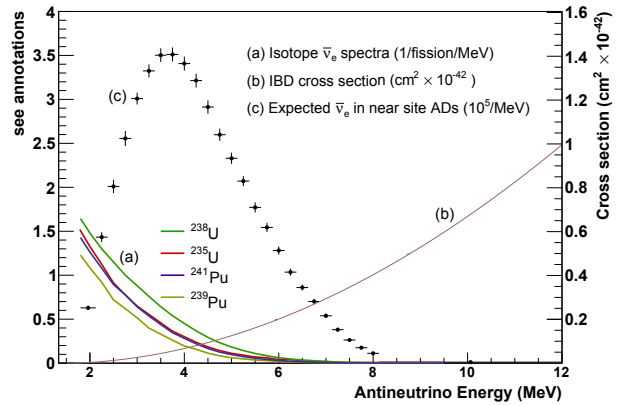

Figure 3: (a) The antineutrino spectra for four isotopes in Huber+Mueller model.(b) The inverse beta decay (IBD) cross section. (c) The expected antineutrino spectrum weighted by the IBD cross section without oscillation in the near site ADs. The error bars are systematic only.

The antineutrinos are detected via inverse beta decay (IBD) interactions: $\bar{v}_{e}+p \rightarrow e^{+}+n$ in the gadolinium-doped liquid scintillator (GdLS) of the Daya Bay ADs. The expected antineutrino spectrum in detector $d$ is calculated by:

$$
S_{d}\left(E_{v}, t\right)=\sum_{r} \frac{1}{4 \pi L_{d r}^{2}} \frac{d^{2} \phi(E, t)}{d E d t} \epsilon^{d} N_{p}^{d} \sigma\left(E_{v}\right),
$$

where $L_{d r}$ is the baseline from reactor $r$ to detector $d, \epsilon^{d}$ is the detection efficiency of detector $d, N_{p}^{d}$ is the target proton number of detector, and $\sigma\left(E_{v}\right)$ is the IBD cross section. Fig. 3 shows the expected antineutrino spectrum without oscillation in the near site ADs.

The inverse beta decay candidates were selected by applying a series cuts, requiring a time coincidence between a prompt signal from the positron and a following delayed signal from the neutron captured on Gd $30 \mu s$ later on average. The selection and cuts are described in [15]. The IBD selection efficiency was evaluated by carrying out detailed Mote Carlo simulation using GEANT4 based simulation package. By considering the IBD selection criteria and effects of target proton number, an absolute detection efficiency common to all detectors was defined and was evaluated to be $80.6 \%$. The total systematic uncertainty of detection efficiency is $1.93 \%$.

The data taking has two periods, during the first period from December 2011 to July 2012, six ADs were deployed for data taking, two ADs were at Daya Bay site(EH1), one at the Ling Ao site(EH2), and three were at the far site (EH3). During the second period from October 2012 to November 2013, the full eight-AD detector configuration was deployed with two ADs at each near sites and four ADs at the far site. With 621 days data taking time, more than 1.2 million inverse beta decay (IBD) candidates were collected, forming the largest reactor antineutrino sample.

\section{Measurement of Reactor Antineutrino Flux}

The reactor flux can be measured by the near site ADs. A normalization factor $R$ was defined to scale the measured reactor antineutrino rate to the predicted rate. Two methods were used to extract 
the reactor antineutrino flux. In the first method, the value of $R$ together with the $\sin ^{2} 2 \theta_{13}$ were determined by a $\chi^{2}$ fitting using all data of the eight ADs. Fig. 4 shows the daily averaged rate of IBD events per $\mathrm{AD}$ in each experimental halls during the data taking time.

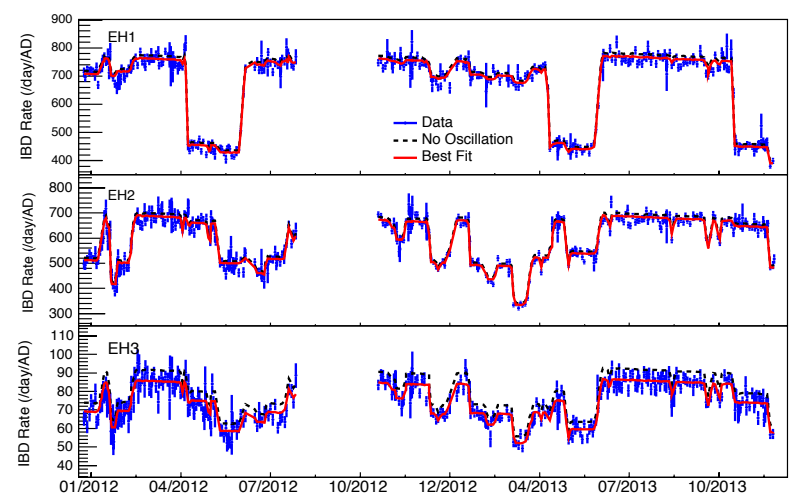

Figure 4: Daily averaged rates of IBD candidate events per AD in the three experimental halls as a function of time. The dotted curves represent no-oscillation predictions based on reactor antineutrino flux analyses and detector simulation. The predictions incorporated the best-fit normalization parameter $(R)$. The red solid curves represent the rates predicted with the best-fit $\sin ^{2} 2 \theta_{13}$.

The $\chi^{2}$ function using the integrated rate information was constructed as below:

$$
\chi^{2}=\sum_{d=1}^{8} \frac{\left[M_{d}-R \cdot T_{d}\left(1+\epsilon_{D}+\sum_{r} \omega_{r}^{d} \alpha_{r}+\epsilon_{d}\right)+\eta_{d}\right]^{2}}{M_{d}+B_{d}}+\sum_{r=1}^{6} \frac{\alpha_{r}^{2}}{\sigma_{r}^{2}}+\sum_{d=1}^{8}\left(\frac{\epsilon_{d}^{2}}{\sigma_{d}^{2}}+\frac{\eta_{d}^{2}}{\sigma_{B, d}^{2}}\right)+\frac{\epsilon_{D}^{2}}{\sigma_{D}^{2}}
$$

where $M_{d}$ is the measured IBD event number in detector $d$ (backgrounds subtracted), $B_{d}$ is the number of background events, $T_{d}$ is the predicted IBD event number by a reactor model with neutrino oscillations, and $\omega_{r}^{d}$ is the fractional IBD contribution from the $r$-th reactor to the $d$-th detector. $\sigma_{r}(0.9 \%)$ is the uncorrelated reactor uncertainty, $\sigma_{d}(0.2 \%)$ is the uncorrelated detection uncertainty, $\sigma_{B, d}$ is the background uncertainty listed in Ref. [16], and $\sigma_{D}$ (1.93\%) is the correlated detection efficiency uncertainty. $\alpha_{r}, \epsilon_{d}, \eta_{d}$, and $\epsilon_{D}$ are the corresponding nuisance parameters respectively.

The minimization of the $\chi^{2}$ function gives the best-fit value of $R$ and $\sin ^{2} 2 \theta_{13}$. The best-fit value of $R$ is $0.946 \pm 0.020(0.992 \pm 0.021)$ with respect to the Huber+Mueller (ILL+Vogel) model. The best-fit value of $\sin ^{2} 2 \theta_{13}=0.085 \pm 0.006$.

In the second method, we use the near site data and correct the oscillation using the value of measured $\sin ^{2} 2 \theta_{13}$. The measured reactor antineutrino flux is expressed in two model-independent ways: the measured IBD events in each detector are normalized to $\sigma_{f}$ with unit: $\mathrm{cm}^{2}$ fission ${ }^{-1}$ and $Y$ with unit: $\mathrm{cm}^{2} G W^{-1} d a y^{-1}$.

$\sigma_{f}$ for each $\mathrm{AD}$ can be determined by solving the following equation:

$$
M_{d}=\sum_{r=1}^{6} \frac{N_{r}^{f}}{4 \pi L_{d r}^{2}} \sigma_{f}^{d} N_{d}^{\mathrm{T}} P_{s u r}^{d r} \epsilon_{d}^{D}
$$




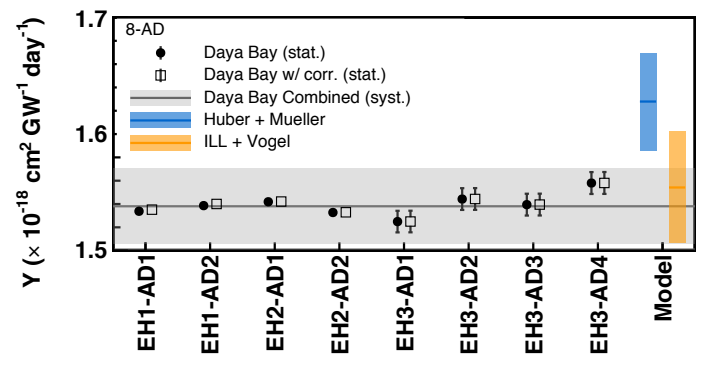

Figure 5. Yield $Y$ for the IBD events with corrections of 3-flavor oscillations (closed circles), and additional corrections to the variations of flux-weighted fission fractions at different sites (open squares). The horizontal line is the average yield of the near detectors, and the gray band is its $1 \sigma$ systematic uncertainty. The rate predicted by the Huber+Mueller (ILL+Vogel) model and its uncertainty are shown in blue (orange) region.

where $N_{r}^{f}$ is the predicted number of fission number of reactor core $r$ using average thermal power and average fission fraction, $N_{d}^{\mathrm{T}}$ is the target proton number, $P_{s u r}^{d r}$ is the survival probability of the antineutrinos from reactor $r$ to $d$, and $\epsilon_{d}^{D}$ is the detection efficiency.

The IBD yield per GW thermal power per day is defined as $Y_{d} \equiv \sigma_{f}^{d} N_{r}^{f} / W_{r}$. Fig. 5 shows the fission yield of the ADs. Table. 1 summaries the IBD fission Yield of the near site ADs, the flux normalization $R$ with respect to Huber+Muller (ILL+Vogel) Model, and the average fission fraction.

Table 1: The average IBD yields $\left(Y\right.$ and $\left.\sigma_{f}\right)$ of the near halls, the flux normalization with respect to different reactor model predictions, and the flux-weighted average fission fractions of the near halls.

\begin{tabular}{c|c}
\hline \multicolumn{2}{c}{ IBD Yield } \\
\hline$Y\left(\mathrm{~cm}^{2} / \mathrm{GW} /\right.$ day $)$ & $(1.53 \pm 0.03) \times 10^{-18}$ \\
$\sigma_{f}\left(\mathrm{~cm}^{2} /\right.$ fission $)$ & $(5.91 \pm 0.12) \times 10^{-43}$ \\
\hline \multicolumn{2}{c}{ Data / Prediction } \\
\hline$R$ (Huber+Mueller) & $0.946 \pm 0.020$ (exp.) \\
$R$ (ILL+Vogel) & $0.992 \pm 0.021$ (exp. $)$ \\
\hline${ }^{235} \mathrm{U}:{ }^{238} \mathrm{U}:{ }^{239} \mathrm{Pu}:{ }^{241} \mathrm{Pu}$ & $0.561: 0.076: 0.307: 0.056$ \\
\hline
\end{tabular}

We also performed a global fit of the past reactor neutrino experiments, comparing the measurements with prediction using Huber+Muller model as the reference, including 21 short baseline measurements using the data from [17] and CHOOZ[18] and Palo Verde [19] . The final result is:

$$
R_{\text {past }}=0.942 \pm 0.009(\text { exp. }) \pm 0.023(\text { model }) \text {, }
$$

The Daya Bay result $R_{D Y B}=0.946 \pm 0.020(\exp$.) is consistent with this global average. The results of the global fit and the Daya Bay measurement are shown in Fig. 6.

\section{Measurement of Reactor Antineutrino Spectrum}

We extend our study from the reactor antineutrino flux to the energy spectrum. The measured positron spectrum of IBD events from the four ADs of the near sites were combined and compared with predictions using Huber+Muller model. The predicted antineutrino energy spectrum was transformed into prompt energy spectrum utilizing the detector response matrix. The response matrix was built considering the IBD prompt energy shift, the effects of energy loss in the inactive acrylic vessels, the non-linearity effect, and the energy resolution. The response matrix was evaluated with two methods: 

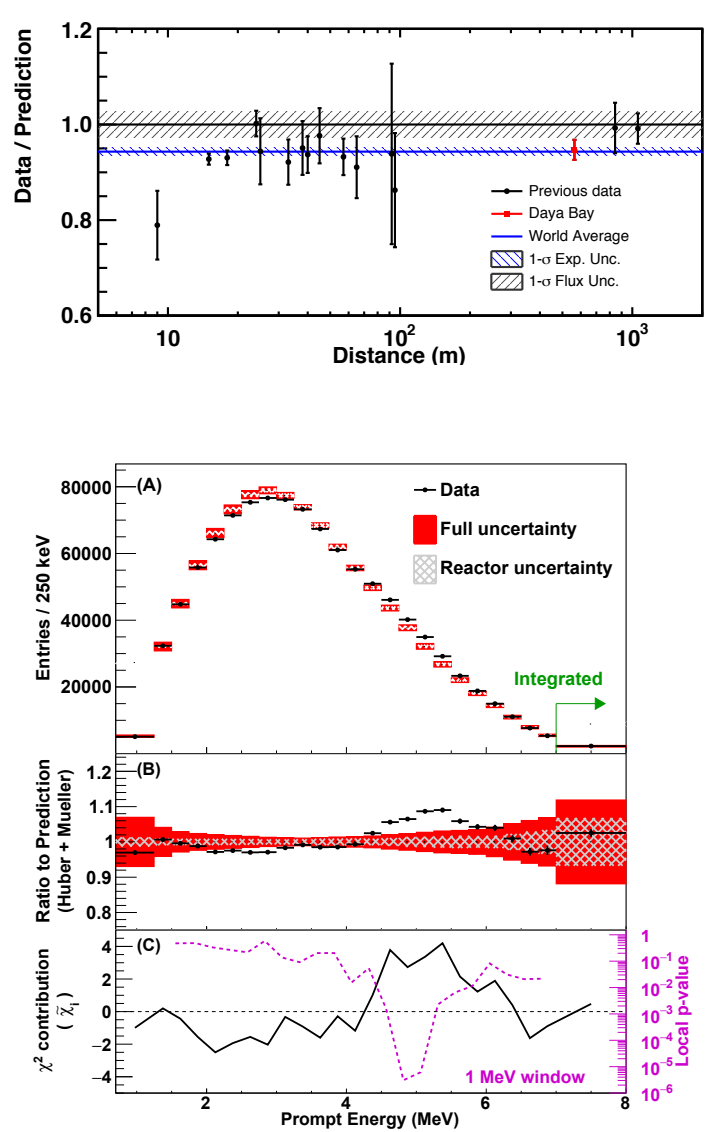

Figure 6. The measured reactor $\bar{v}_{e}$ rate as a function of the distance from the reactor, normalized to the prediction using Huber+Mueller model. The rate is corrected with neutrino oscillations at the distance of each experiment. The purple shaded region represents the global average and its $1 \sigma$ uncertainty. The $2.4 \%$ model uncertainty is shown as a band around unity. The measurements at the same baseline are combined together for clarity. The Daya Bay measurement is shown at the flux-weighted baseline $(573 \mathrm{~m})$ of the two near halls.

Figure 7. (A) Comparison of predicted and measured prompt energy spectra. The error bars on the data points represent the statistical uncertainty. The hatched and red filled bands represent the square-root of diagonal elements of the covariance matrix $\left(\sqrt{\left(V_{i i}\right)}\right)$ for the reactor related and the full systematic uncertainties, respectively. (B) Ratio of the measured prompt energy spectrum to the predicted spectrum. (C) The defined $\chi^{2}$ distribution $\widetilde{\chi}_{i}$ ) of each bin (black solid curve) and local P-values for $1 \mathrm{MeV}$ energy windows (magenta dashed curve).

a step-by-step analytical method and a full detector simulation method base on GEANT4. Both methods produced consistent results. Fig. 7 shows the measured near site combined prompt spectrum and its comparison with the prediction. The prediction based on the Huber+Mueller model is normalized to the number of measured events.

From Fig. 7 , a "bump" like structure is clearly visble. A $\chi^{2}$ function was constructed to qualify the discrepancy of the measurement and the prediction:

$$
\chi^{2}=\sum_{i, j}\left(N_{i}^{\mathrm{obs}}-N_{i}^{\mathrm{pred}}\right)\left(V^{-1}\right)_{i j}\left(N_{j}^{\mathrm{obs}}-N_{j}^{\mathrm{pred}}\right)
$$

where $N_{i}^{\mathrm{obs}(\text { pred) }}$ is the observed (predicted) number of events at the $i$-th prompt energy bin and $V$ is the covariance matrix that including all uncertainties. The energy ranges from 0.7 to $12 \mathrm{MeV}$. Fitting the $\chi^{2}$ function yields $\chi^{2} / d o f=46.6 / 24$, corresponding to a $2.9 \sigma$ discrepancy over all the energy range.

To qualify the local discrepancy of the bump structure, another two methods were deployed to evaluate the significance of the deviation. The first method calculates the $\chi^{2}$ contribution of each 
energy bin:

$$
\begin{aligned}
\tilde{\chi}_{i} & =\frac{N_{i}^{\mathrm{obs}}-N_{i}^{\mathrm{pred}}}{\mid N_{i}^{\mathrm{obs}}-N_{i}^{\mathrm{pred}}} \mid \sqrt{\sum_{j} \chi_{i j}^{2}}, \\
\chi_{i j}^{2} & =\left(N_{i}^{\mathrm{obs}}-N_{i}^{\mathrm{pred}}\right)\left(V^{-1}\right)_{i j}\left(N_{j}^{\mathrm{obs}}-N_{j}^{\mathrm{pred}}\right) .
\end{aligned}
$$

From Fig. 7 panel (C), the enhanced contribution around $5 \mathrm{MeV}$ can be seen clearly. The second method introduces $\mathrm{N}$ (number of bins) nuisance parameters with no pull terms to the oscillation fitter. The $\chi_{\min }^{2}$ difference before and after introducing the $\mathrm{N}$ nuisance parameters is expected to follow a $\chi^{2}$ distribution with N-1 d.o.f., therefore a P-value can be calculated. Fig. 7 panel (C) shows the P-values with $1 \mathrm{MeV}$ sliding energy window, and around $5 \mathrm{MeV}$ the discrepancy is $>4 \sigma$. Further more, the local significance for the $2 \mathrm{MeV}$ window between 4 and $6 \mathrm{MeV}$ was evaluated, and we obtained $\Delta \chi^{2} /$ dof $=37.4 / 8$, corresponding to the P-value of $9.7 \times 10^{-6}(4.4 \sigma)$. The events around 5 $\mathrm{MeV}$ were carefully examined, the events match all IBD event characteristics, more checks ruled out detector effects, simple background contribution and other interaction channels as explanations. This study suggests that the deviation is due to the imperfection of the modeling of the reactor antineutrino spectrum.

A generic reactor antineutrino spectrum for the IBD reaction was extracted from the Daya Bay measured spectrum. This spectrum is independent with the Daya Bay detector response and could be used as an input to predict the reactor antineutrino flux and spectrum for other experiments. The near site measured prompt spectrum were combined together, and then the detector response effects were removed by utilizing unfolding to the prompt spectrum to obtain the antineutrino spectrum. Two unfolding algorithms, the Singular Value Decomposition(SVD) and Bayes iteration algorithms were applied and got almost same results. In addition, the independence of unfolded spectrum to Monte Carlo input of response matrix, the minimization of variance plus bias were all tested.

Oscillation effects was then removed from the unfolded antineutrino spectrum and further more each energy bin of the antineutrino spectrum was normalized to $\mathrm{cm}^{2} /$ fission $/ \mathrm{MeV}$ using the reactor information, making the antineutrino spectrum to be a model-independent spectrum:

$$
S_{\text {generic }}(E)=\frac{S_{\text {combined }}(E)}{P_{\text {sur }}(E, L) \cdot N_{P} \cdot F_{\text {total }}}
$$

where $S_{\text {combined }}$ is the combined IBD prompt spectrum of the near site ADs, $P_{\text {sur }}(E, L)$ is the average survival probability of the $\bar{v}_{e}$ calculated with the fluxes from the six reactors to the four detectors, $N_{P}$ is the number of target protons of the average target mass, and $F_{\text {total }}$ is a normalization factor based on the baseline-weighted total number of fissions. Fig. 8 shows the obtained generic antineutrino spectrum. As a comparison, the predicted spectrum of the near site antineutrino spectrum was processed with the same steps as in Equation (8) and was plotted in Fig. 8 Panel (B), which has the same rate deficit as the flux measurement and similar spectral deviations as in the comparison of measured and predicted IBD prompt spectra.

A possible application of the generic spectrum is to predict antineutrino spectrum for other reactor experiments. A simple example is shown below. Suppose a reactor antineutrino experiment A, the predicted antineutrino spectrum of experiment A can be expressed as:

$$
S_{A}=S_{d y b}+\sum_{i}\left(f_{A_{i}}-f_{d y b_{i}}\right) S_{\bmod _{i}}
$$

where $i$ is the index of the four fissile isotopes, $S_{d y b}$ is the Daya Bay generic spectrum $S_{\text {generic }}(E)$, $f_{d y b}$ and $f_{A}$ are the average fission fractions of the Daya Bay experiment and the reactor antineutrino 


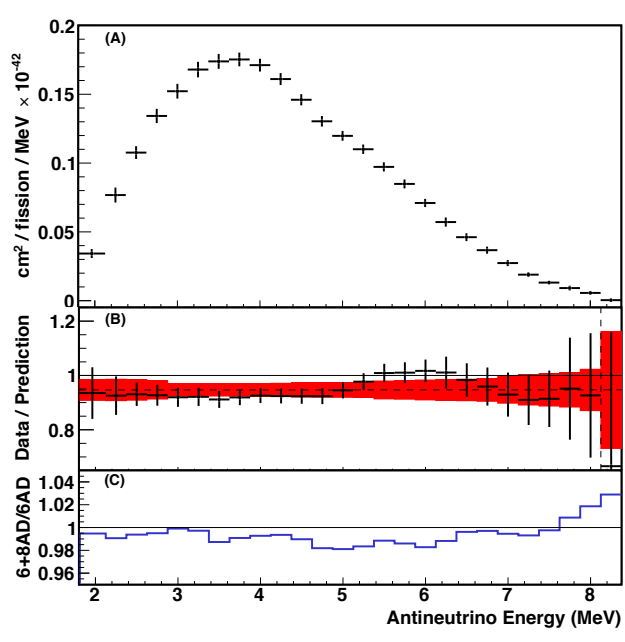

Figure 8. (A) The antineutrino spectrum weighted by the IBD cross section. The last bin is integrated up to $12 \mathrm{MeV}$. (B) Ratio of the extracted reactor antineutrino spectrum to the Huber+Mueller prediction. The error bars of the data points are the square-roots of the diagonal elements of the antineutrino spectrum covariance matrix. The solid red band represents the square-roots of the diagonal elements of the prediction covariance matrix, including both reactor and Huber+Mueller model uncertainties. (C) the ratio of the spectra from the $6+8 \mathrm{AD}$ periods used in this analysis and the $6 \mathrm{AD}$ period used in the previous analysis [20].

experiment $A$, and $S_{m o d}$ are the isotope antineutrino spectra from models, such as ILL+Vogel, Huber+Mueller, etc. This method works when the difference of the average fission fraction between experiment A and Daya Bay is relative small, which is usually true for PWR reactor experiments.

\section{Summary}

With 621 days of data taken by eight ADs, more than 1.2 million IBD events were collected by the Daya Bay experiment. The measured absolute reactor antineutrino flux is consistent with previous measurements. The measured IBD yield is $(1.53 \pm 0.03) \times 10^{-18} \mathrm{~cm}^{2} / \mathrm{GW} /$ day or $(5.91 \pm 0.12) \times$ $10^{-43} \mathrm{~cm}^{2} /$ fission. The ratio of measured flux to the predictions is $0.946 \pm 0.020(0.992 \pm 0.021)$ for the Huber+Mueller (ILL+Vogel) model. The predicted and measured spectra were compared and found a deviation of $2.9 \sigma$. In the region of 4-6 MeV the event excess has a local significance of $4.4 \sigma$. A generic reactor antineutrino spectrum was extracted from the measured IBD prompt spectrum, providing a model-independent prediction option for future reactor antineutrino experiments.

\section{Acknowledgement}

Part of work in this paper is supported by the Fundamental Research Funds for the Central Universities (Program No.222201414032), and by the National Natural Science Foundation of China (11405057, 11390383).

The Daya Bay Experiment is supported in part by the Ministry of Science and Technology of China, the United States Department of Energy, the Chinese Academy of Sciences, the CAS Center for Excellence in Particle Physics, the National Natural Science Foundation of China, the Guangdong provincial government, the Shenzhen municipal government, the China General Nuclear Power Group, the Research Grants Council of the Hong Kong Special Administrative Region of China, the Ministry of Science and Technology and the Ministry of Education in Taiwan, the U.S. National Science Foundation, the Ministry of Education, Youth and Sports of the Czech Republic, the Joint Institute of Nuclear Research in Dubna, Russia, the NSFC-RFBR joint research program, the National Commission for Scientific and Technological Research of Chile. We acknowledge Yellow River Engineering Consulting Co., Ltd. and China Railway 15th Bureau Group Co., Ltd. for building the underground laboratory. 
We are grateful for the ongoing cooperation from the China Guangdong Nuclear Power Group and China Light \& Power Company.

\section{References}

[1] F. Reines, et al., Phys. Rev. 117 (1960) 159.

[2] F. P. An et al. (Daya Bay Collaboration), Phys. Rev. Lett. 108 (2012) 171803

[3] K. Abe et al. (T2K Collaboration), Phys. Rev. D 88, 032002 (2013).

[4] P. Adamson et al. (MINOS Collaboration), Phys. Rev. Lett. 110, 171801 (2013).

[5] Y. Abe et al. (Double Chooz), Phys. Rev. Lett. 108, 131801 (2012).

[6] J. K. Ahn et al. (RENO), Phys. Rev. Lett. 108, 191802 (2012).

[7] K. Schreckenbach, G. Colvin, W. Gelletly, and F. Von Feilitzsch, Phys.Lett. B160, 325 (1985).

[8] F. Von Feilitzsch, A. A. Hahn, and K. Schreckenbach, Phys.Lett. B118, 162 (1982).

[9] A. A. Hahn et al., Phys.Lett. B218, 365 (1989).

[10] P. Vogel, G. K. Schenter, F. M. Mann, and R. E. Schenter, Phys.Rev. C24, 1543 (1981).

[11] T. A. Mueller et al., Phys.Rev. C83, 054615 (2011).

[12] P. Huber, Phys.Rev. C84, 024617 (2011).

[13] R. Sanchez et al., Nucl. Eng. Tech. 42, 474 (2010).

[14] X. B. Ma et al., Phys.Rev. C88, 014605 (2013).

[15] F. P. An et al. (Daya Bay Collaboration), Chin.Phys. C37, 011001 (2013).

[16] F. P. An et al. (Daya Bay Collaboration), Phys. Rev. Lett. 115, 111802 (2015).

[17] G. Mention et al., Phys.Rev. D83, 073006 (2011).

[18] M. Apollonio et al., Phys.Lett. B466, 415 (1999).

[19] F. Boehm et al., Phys.Rev. D64, 112001 (2001).

[20] F. P. An et al. (Daya Bay Collaboration), Phys. Rev. Lett. 116, 061801 (2016). 